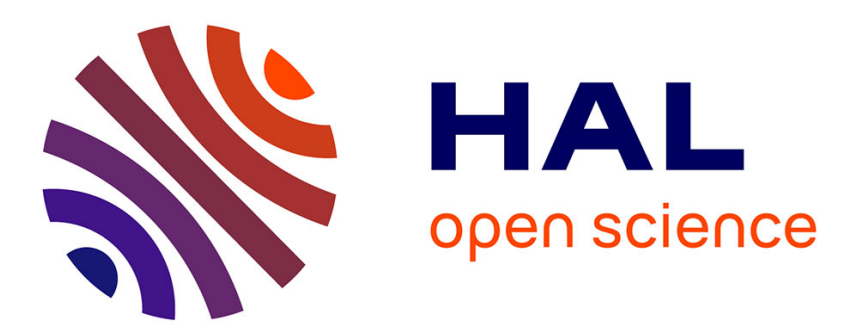

\title{
L'accompagnement de la construction de la chaîne associative groupale dans un groupe de patients schizophrènes
}

\author{
Guy Gimenez, Jean-Pierre Pinel, Claudine Vacheret
}

\section{- To cite this version:}

Guy Gimenez, Jean-Pierre Pinel, Claudine Vacheret. L'accompagnement de la construction de la chaîne associative groupale dans un groupe de patients schizophrènes. Bulletin de psychologie, 2013, Psychologie clinique des groupes et des médiations thérapeutiques, de l'institution et du lien social, 526, pp.303-308. 10.3917/bupsy.526.0303 . hal-01384266

\section{HAL Id: hal-01384266 \\ https://hal-amu.archives-ouvertes.fr/hal-01384266}

Submitted on 19 Oct 2016

HAL is a multi-disciplinary open access archive for the deposit and dissemination of scientific research documents, whether they are published or not. The documents may come from teaching and research institutions in France or abroad, or from public or private research centers.
L'archive ouverte pluridisciplinaire HAL, est destinée au dépôt et à la diffusion de documents scientifiques de niveau recherche, publiés ou non, émanant des établissements d'enseignement et de recherche français ou étrangers, des laboratoires publics ou privés. 


\section{l'accompagnement de la constructionde la chaîne associative groupale dans un groupe de patients schizophrènes}

\author{
Gimenez Guy* \\ PINEL Jean-Pierre** \\ VACHERET Claudine ${ }^{* * *}$
}

Dans ce travail, nous poursuivons quatre objectifs. Nous souhaitons tout d'abord étudier, à partir de séquences cliniques d'un groupe de patients schizophrènes, le processus de construction de chaîne associative groupale (Kaës, 1994), dans son double mouvement de liaison et de déliaison psychique. Sera mis en évidence comment les productions morcelées et dissociées de patients schizophrènes, délirants (et hallucinés) en situation de groupe, peuvent, dans certaines conditions (que nous décrirons), devenir progressivement une chaîne associative de groupe, articulant et nouant les productions individuelles et groupales.

Nous voudrions également étudier comment ces patients, relativement régressés, sont pourtant aptes à « prêter à l'autre » des fonctions psychiques non disponibles pour eux-mêmes (comme, par exemple, la figuration, la scénarisation, etc.). Il s'agit d'une fonction phorique, au sens de René Kaës (1993; 1994), particulière, le groupe devenant un lieu de mise en commun et de partage, une « coopérative psychique » de fonctions non disponibles aux sujets eux-mêmes.

Un intérêt particulier sera porté aux interventions spécifiques du clinicien en groupe, les « interventions double face »(Gimenez, 1996, 2003), traduisant le vécu de la séance, à la fois, au niveau individuel et groupal, au niveau d'un mode de fonctionnement archaïque et plus élaboré. Nous étudierons comment ces interventions peuvent avoir, dans le groupe, la fonction d'articulateurs relationnel-émotionnel pour chaque participant, organisateur intra et inter-psychique, qui rend possible la construction, l'organisation et l'intégration des chaînes associatives individuelles dans une chaîne associative groupale (Kaës, 1994) en train de se constituer.

Nous souhaitons, enfin, décrire une partie du travail interne du clinicien, en particulier le repérage de ce que Gimenez (2000) a nommé «les scénarios de base », c'est-à-dire les scénarios énoncés dans le groupe et épurés des personnages et compléments qui le qualifient et habillent le scénario. Ces scénarios sont structurés comme une phrase : sujet, verbe, complément, sous la forme : « quelqu'un fait quelque chose à quelqu'un d'autre, éventuellement devant un troisième, qui regarde ». Il s'agira d'observer leur succession et leur articulation chez chaque sujet et dans le groupe, dans une séance et entre les séances. Le clinicien aura à élaborer ces liens, et en quoi ces scénarios de base figurent ou tentent de figurer ce qui se passe chez les sujets et dans le groupe.

\section{PRÉSENTATION DU GROUPE}

Nous présenterons maintenant une séquence clinique d'un groupe de parole, constitué de patients schizophrènes. Ce groupe a été mis en place dans un centre hospitalier spécialisé, dans une unité de soins de réadaptation. Il s'agit de patients qui, pour la plupart d'entre eux, n'avaient pas réussi à sortir de l'hôpital de façon durable depuis de longues années et pour lesquels une sortie semblait impensable. Une partie des patients a une pathologie lourde, productive, délirante et souvent accompagnée d'hallucinations. Il s'agit d'un groupe hebdomadaire semi-ouvert, constitué, à la période que nous allons présenter, de sept patients schizophrènes, accompagnés par l'un d'entre nous, psychanalyste de groupe ${ }^{1}$. Les séances durent une heure et demie. La règle de la libre association (on laisse venir ses pensées et on les traduit en mots), dans le respect de chacun, la règle de la discrétion

* Laboratoire Psychopathologie clinique, langage et subjectivité LPCLS (EA 3278), Université Aix-Marseille.

** Unité transversale de recherche : Psychogenèse et psychopathologie (UTRPP - EA 4403), Université Paris 13.

*** Centre de recherche en psychopathologie et psychologie clinique (CRPPC), Université Lumière Lyon 2.

Correspondance : Guy Gimenez, Université AixMarseille, UFR de psychologie, 29 avenue Robert Schuman 13621 Aix-en-Provence cedex 1.

<guy.gimenez@univ-amu.fr>

1. Nous le désignerons indifféremment par le terme de clinicien ou de psychanalyste. 
(ce qui est dit dans le groupe reste dans le groupe) sont énoncées de façon explicite au début du groupe. Chacun s'engage à participer de façon assidue aux séances.

\section{CHAÎNE DISSOCIATIVE ET POINTILLÉS ASSOCIATIFS}

Nous avons choisi la $37^{\mathrm{e}}$ rencontre, qui a été, pour plusieurs patients, un début de reprise élaborative d'expériences traumatiques importantes. Le clinicien a vécu cette séance comme très chargée émotionnellement. Chacun s'assied et le groupe commence par un moment de silence.

\section{Première partie de la séance : l'éparpillement}

Rachida (40 ans), assise au bord de sa chaise, est comme toujours outrageusement maquillée. Elle semble avoir du mal à tolérer ce court moment de silence. Elle scrute, avec tension, le regard de chacun, puis dit : " J'ai gagné au loto... à Matignon j'étais au gouvernement, le directeur de l'hôpital est amoureux de moi, c'est un beau garçon, je vais bientôt passer à la télévision ». Nous repérons le scénario de base : "un homme (important) aime une femme (célèbre) ».

Isabelle (45 ans), assez forte, enserrée dans des vêtements visiblement trop petits pour elle, e1 provocateurs, semble très préoccupée à essayer, en vain, d'ajuster son chemiser laissant apparaître un décolleté très ouvert et sa jupe bien trop courte. Son visage est livide, figé, et sans aucune expression. Elle dit, sans lien apparent avec ce que vient de dire Rachida : «Je fais attention à mon poids, parce que mon ventre est infecté, je vais demander des lavements au docteur... J'ai attrapé la maladie du chien : j'ai les cheveux et les poils comme les chiens, je me mets à quatre pattes comme les chiens, mon mari aime bien... et me prend en levrette. Il paraît que ça se soigne avec le Dogmatil »... Le clinicien entend, en écho, le scénario de base : « une femme est malade (de la maladie du chien)», «un homme prend une femme ».

Philippe (27 ans), habillé de façon négligée, le regard hagard, fait des mouvements rythmiques avec son pied droit, comme s'il essayait d'attraper quelque chose sur le sol, de façon insistante. Il reprend, comme à chaque début de séance, une ritournelle, à mi-voix, qui semble l'apaiser : « Je positive et je négative, je positive et je négative, je positive et je négative, je positive et je négative ». Cette expression répétitive semble avoir, pour lui, l'effet d'une berceuse ou d'un auto-bercement rythmique enveloppant (Avron, 1996) et n'agace plus le groupe, comme au début, mais, au contraire, l'enveloppe, comme un fond sonore apaisant (enveloppe sonore). Nous repérons, ici, le signifiant formel (Anzieu, 1987) : « un corps se berce (est bercé) répétitivement ».

Gérald (27 ans), reprend, de façon un peu décalée : "J'ai bien peur qu'ils aient mis ma fille sur le trottoir ou qu'ils l'aient tuée... Ici on me drogue. Mon hospitalisation est un coup monté de ma belle famille. Ils sont en connivence avec une secte puissante et dangereuse, tout ça pour m'éloigner de mes enfants. » Le superviseur interne du clinicien (Casement, 1985) traduit: " On fait du mal (on prostitue, on tue) un enfant (éloigné) ».

Isabelle reprend, sans lien apparent: "Vous trouvez pas que la soupe avait un drôle de goût hier soir ? J'ai peur... ». Nous repérons qu'elle reprend à son compte la dimension persécutive induite par Gérald.

Catherine (41 ans), assise à côté d'elle, a, comme très souvent, de façon très énigmatique, la tête tournée à 90 degrés comme les statues pharaoniques. Elle reste silencieuse, imperturbable, les yeux écarquillés, mais son regard semble perdu au loin, dans son univers intérieur, gardé hermétiquement secret.

Isabelle reprend la thématique sexualisée et persécutive, à propos de son corps : «Pour mon accouchement j'ai dû me mettre toute nue. Et on m'a mis une longue tige dans le sexe, comme pour m'empaler. Et j'ai eu une hémorragie interne. Ils m'ont fait une piqure dans le ventre, ça m'a rendue stérile. Puis ils m'ont fait une piqûre dans les fesses et ça m'a rendue folle. C'est pour ça que je suis ici. ». Gérald sourit sans rien dire. Le psychanalyste est touché par le récit d'Isabelle, qui, malgré sa dimension délirante, tente de mettre en forme, autour d'un signifiant formel (un corps est pénétré, abîmé), ce qui s'est passé pour elle, lors de sa décompensation, juste après son accouchement (psychose puerpérale).

Philippe dit alors : «Je positive et je négative, je positive et je négative, je suis un humain quand même $» .$.

Éric reprend les derniers mots de Philippe : «Des êtres humains... À l'école, j'écoutais mais je ne comprenais rien, c'était comme une langue étrangère... Et les gens que je croisais dans la rue avaient mon visage » (figures du double).

\section{Commentaire}

Ce propos est ressenti par le clinicien ressent comme une figuration de ce qui se passe en lui : cette impression troublante de ne pas réussir à comprendre vraiment ce qui se passe dans le groupe, et la langue de chacun (babélisation). Dans ce premier temps, le psychanalyste entend bien les mots, les phrases et les scénarios de base, mais il reste en partie sourd au sens de leur succession, trop rapide, et à leur articulation : « un homme aime 
une femme »; « une femme est malade»; « un corps est bercé »; « on fait du mal ou éloigne des enfants »; « un corps est pénétré », « une femme change d'identité ». Il ressent de nombreux éléments en suspens, comme dans la position paranoïde-schizoïde décrite par Melanie Klein (1946). Le clinicien se sent éparpillé, débordé, saturé, rempli d'un en-trop émietté. Il se sent envahi par une fatigue, qui semble liée à l'immense énergie utilisée pour garder ensemble et relier ce qui, de toute évidence, ne s'articule pas assez pour sa psyché, bien trop en quête de sens.

Les éléments associatifs co-présents semblent interpénétrés, conglomérés, compactés, non articulés, non organisés, et la tentative du psychologue, pour les relier en lui-même, augmente ce sentiment de déliaison et d'éparpillement, comme si la tentative de travail de lien en révélait son négatif actif : l'attaque et la destructivité du lien, du sens, de la signification. C'est ici, nous en émettons l'hypothèse, une réaction contre-transférentielle groupale à ce premier temps de mise en dépôt, en moi et dans le groupe, de ce qui ne peut rester paisiblement dans le dedans de chacun des participants, écho des parts dissociées de chacun.

Nous comprenons, après-coup, une part de ce vécu comme une expression du «devenir $\mathrm{O}$ » (Bion, 1970, p. 63) en groupe (Gimenez, 2003), décrite par Bion, dans sa dimension régressive et non liée : transformation de l'être par et dans la présence des autres, connectés à leurs noyaux les plus primitifs et, peut-être, dans une mobilisation particulière de notre groupe interne archaïque (Kaës, 1993 ; 1999), laissant la parole à des parts de soi, plus ou moins autonomes, qui d'habitude demeurent cachées et silencieuses au fond de chacun.

Le clinicien dit: "Parfois on écoute et on ne comprend pas, on s'écoute et on ne se comprend pas vraiment, on peut ne pas se comprendre soimême ou certaines parties de soi, on peut ne pas comprendre les autres; ça peut, en effet, être comme une langue étrangère qu'il nous faut apprendre. Ici, dans le groupe, on peut, peut-être, ensemble, apprendre à s'écouter, soi-même et les autres, à traduire ce que l'on dit, et ce que les autres disent ».

\section{Deuxième partie de la séance}

Gérald dit alors : «Ma mère buvait de trop, ça l'a rendue folle. Elle me frappait à coup de bouteilles, elle me giflait, me traînait par les cheveux, me frappait à coup de poings... Mon père n'était jamais à la maison, il était toujours en déplacement, il était chauffeur de camions, j'étais terrorisé. J'avais peur qu'elle meure ». Le clinicien est touché par le fait que Gérald puisse parler de sa souffrance intense, même s'il ne parvient pas à parler ni à penser ses mouvements hostiles et haineux envers sa mère maltraitante, et le clinicien ne dit rien. Son superviseur interne (Casement, 1985) traduit : « une mère maltraite un enfant » et, en écho, «un enfant protège sa mère ».

Rachida, visiblement inquiète et touchée par ce que vient de dire Gérald, éclate de rire (défense maniaque) et dit: "Je suis la plus belle fille du monde, il me faut des gardes du corps ».

Pensant à ce que vient de déposer Gérald dans le groupe et l'association, à moitié défensive, à moitié figurative, de Rachida, il reprend: «Des fois on a peur et on a besoin d'être protégé, de ses peurs, de ce qui pourrait nous arriver ou arriver à ceux qu'on aime, et on peut aussi avoir peur de ce qu'on pourrait faire...».

Après un moment de silence, Jean-Paul, qui est arrivé récemment dans le groupe, prend la parole et dit, de façon apparemment un peu décalée : «Je voudrais un jour de plus pour ma permission ». Il précise, au bout d'un moment: «Pour voir ma mère ». Isabelle, qui est souvent très délirante et hors réalité le recadre pourtant: "Ici c'est un groupe de parole, pour tes permissions, tu dois en parler avec le médecin ». Le clinicien est, une fois de plus, touché de voir combien les patients schizophrènes, par ailleurs très dissociés, peuvent « porter pour les autres ce qu'ils ne peuvent pas forcément faire pour eux-mêmes («porte processus » fonctions phoriques spécifiques, telles qu'elles sont définies par René Kaës), chacun peut ainsi «prêter aux autres » et dans le groupe, des fonctions psychiques qui ne semblent pourtant pas disponibles pour eux, dans leur vie quotidienne (fonction de coopérative psychique).

Rachida reprend (en haussant les épaules) : «Ma mère, elle, ne s'occupe pas de moi, elle ne vient pas me voir à l'hôpital. Quand je délirais, je suivais la violence de Dieu et la sagesse du diable. Pourriez-vous m'aider à retrouver mes parents ? J'ai le nom de tous ceux qui me font du mal».

Éric reprend à son tour : «J'ai été enlevé par les indiens d'Amazonie et j'ai été élevé par une mère cannibale. Elle m'a protégée et elle me déguisait en fille. Des fois, j'ai des idées noires, je pense à tuer ma mère. Ma mère je l'aime plus que moimême ». (Il met sa tête entre ses mains, visiblement bouleversé par ce qui se passe en lui et dans le groupe). Chacun semble troublé par ce qui vient d'être dit, d'autant qu'Éric sort d'une unité de malades difficiles, où il est allé après des passages à l'acte graves sur des membres de sa famille. Nous faisons l'hypothèse qu'Éric figure, en partie de façon délirante, les deux facettes du lien à la mère, dont seule la part attaquante et maltraitante avait pu être exprimée par Gérald et Rachida. Nous 
entendons un scénario emboîté du type : «Une mère (dangereuse) protège un enfant (en danger) ». Et dans un scénario faussement ambivalent : «Un enfant aime sa mère, un enfant veut tuer sa mère ».

Christiane reprend, comme en écho : « Ma mère disait que je n'étais pas sa fille. Elle me battait. Je suis la fille du frère au mari à ma mère... mon oncle a violé ma mère... et ma mère m'a laissée à l'assistance publique... ». Un silence intense s'installe dans le groupe. «Ma mère est morte en chambre d'isolement après y avoir mis le feu, c'est ce que le médecin a dit... ». Résonne violemment, à l'intérieur du psychanalyste, le scénario: une mère (maltraitante et abondonnante) est attaquée, violée. Une mère meurt.

Il sent son ventre se nouer, saisi par la dimension traumatique de ce que vient de dire Christiane, et qu'il sait, par ailleurs, ne pas être délirant. Tout semble se passer comme si la succession des associations des membres du groupe rendait progressivement possible la figuration d'un vécu traumatique intolérable et quasi-impensable. Le clinicien essaie de se replacer en position «méta », autoobservateur et penseur de ce qui se passe..., mais en vain. Il se sent intensément en lien avec la souffrance de Christiane, de Gérald, d'Éric et celle du groupe. Il ressent, pendant quelques secondes (une éternité), une sensation d'effroi et de vide sidérant, glaçant, quasi-intolérable, qu'aucun de ses mots ne pourrait traduire ; pas une absence de substance ou de présence, mais le résultat (destructeur) d'absorption, consistance de non présence, tout l'être aspiré vers l'extérieur de lui-même, comme un vidage dans un dedans inexistant, et sans enveloppe (signifiant formel : « un corps se vide dans le vide»).

Il retrouve une sensation très désagréable et douloureuse de cauchemars de l'enfance, de tomber dans un trou sans fond et sans mains pour retenir, tenir, porter... Il se sent abandonné par son superviseur interne, qui ne l'aide pas à relier ce qui vient d'être dit dans le groupe. Silencieux, et envahi par ces sensations, il ne trouve pas (assez vite) les mots pour traduire ce qu'il vit pour lui-même et pour le groupe...

Catherine, qui avait gardé, jusque-là, sa tête à 90 degrés, la redresse légèrement et dit : «Je suis la reine du Brésil. J'ai été kidnappée dans un réseau de prostitution. Je suis une princesse... J'ai arrêté la bombe à retardement qui devait détruire la terre. N'oubliez pas, il y a une bombe à retardement. Est-ce que les feuilles d'arbres ont repoussé ? ».

Le psychanalyste se sent aidé et soutenu par l'intervention de Catherine, qui donne forme à cette tension sourde qui l'envahissait, dépôt, en lui et dans le groupe, de ce qui ne pouvait rester au fond de Christiane et de plusieurs autres (fonction phorique, porte figuration). Il dit à Catherine et au groupe : « En effet, il y a parfois, comme plusieurs l'ont dit dans ce groupe, des choses très douloureuses au fond de nous, comme des bombes à retardement, des choses qu'on vit comme si difficiles et si dangereuses, pour nous et pour les autres, qu'on se demande si la vie va pouvoir continuer et reprendre son cours ». Le groupe reste un moment silencieux, comme dans le partage d'émotions, ressenties intensément en commun, en ce moment protégé, de peine partagée.

Et comme pour conclure la séance, pour luimême et pour le groupe, Philippe reprend : « Je positive et je négative, et finalement, j'ai perdu mes rêves... maintenant, je suis parti pour être un homme et je cherche qui je suis. » Et il poursuit : «Le lit de mon enfance n'a pas grandi avec moi. Lui est resté tout petit et moi j'ai grandi tout seul... ». Le patient demande, les yeux humides : «C'est quoi cette solitude que je transporte partout avec moi ? ». Le clinicien sent que Philippe porte, pour le groupe, un mouvement dépressif, lié à un début de travail introjectif sur les mouvements violents, destructeurs et haineux, quasi impensables, du lien à la mère.

Le clinicien regarde Philippe et l'ensemble du groupe et dit : " Quand on grandit, avec des bombes à retardement à l'intérieur de soi, c'est très douloureux, et on peut perdre ses rêves d'enfance, se sentir très seul et chercher qui l'on est. Des choses ou des gens proches manquent autour de soi. Les bras qui nous tenaient ou qu'on a rêvés ne sont plus là, et on peut éprouver une grande peine d'avoir perdu ce qui semblait si bon pour le petit garçon ou la petite fille au fond de nous ».

C'est la fin de la séance.

\section{QUELQUES COMMENTAIRES POUR CONCLURE}

\section{Les pointillés associatifs}

$\mathrm{Au}$ début de la séance, l'attention est centrée sur le repérage des «pointillés associatifs », qui, d'un regard extérieur, semblent former une "chaîne dissociative »: successions d'éléments associatifs individuels juxtaposés, souvent imbriqués dans des constructions délirantes, qui en rendent la compréhension difficile. Ces éléments associatifs semblent épars, éparpillés et parfois éparpillant, car chargés d'une partie des processus dissociatifs (et de morcellements) individuels dont ils sont issus. C'est ainsi que nous appréhendons la cacophonie associative comme une polyphonie potentielle, ce qu'elle peut quelquefois devenir en passant par la psyché du clinicien, dans l'espace du groupe, qui lui fournit un lieu et une enveloppe où loger ces éléments épars et éparpillant (Gimenez, 2006). Nous avons pris le parti, ici, de traiter le 
« morcelé » comme du « diffracté », c'est-à-dire comme bien plus élaboré qu'il ne l'est, par nécessité clinique (Gimenez, 2006). Cette « illusion efficace » rend possible le traitement du chaos non organisé, issu des «pointillés associatifs » individuels interpénétrés, conglomérés, et articulés uniquement en apparence : rêver le lien (au sens de capacité de rêverie) en rend son advenue possible.

\section{Recevoir les maillons associatifs}

Le travail clinique a consisté d'abord à accueillir chacun de ces éléments qui peuvent ainsi cohabiter dans le clinicien et dans le groupe, comme nous l'avons vu. Cet hébergement peut provoquer des effets : saturation, sentiment de trop plein, de débordement, d'éparpillement, de morcellement.

\section{Repérer les scénarios de base}

Mais l'hébergement rend aussi possible le repérage des scénarios de base sous-jacents, afin de saisir en quoi ceux-ci figurent ce que le groupe tente de mettre en forme. Il s'agit ici d'un travail de traduction de ce que le patient dit, pour le groupe et, en retour, pour lui-même. On observe ainsi, le plus souvent, que les patients, sans le savoir, et quelquefois de façon délirante, produisent des éléments de scénarios permettant à la chaîne associative de se construire. Nous traitons les associations individuelles dans le groupe comme porteuses potentielles de signification (Gimenez, 2006). Ce préinvestissement favorise l'utilisation de ces associations comme support possible d'une chaîne associative spécifique (chaîne associative primaire archaïque). Les scénarios de base repérés « appellent » chez le clinicien un travail associatif et de liaison. Mais le travail ne consiste pas ici à re-trouver les liens de sens latents, qu'il faudrait mettre en lumière ou conscientiser, mais d'en tisser, de les construire, de les développer : leur donner forme, figure et consistance. Chacun de ces pointillés associatifs, produits en situation de groupe, sont, pour nous, des tentatives de chaque patient, pour figurer, pour euxmêmes, ce qu'ils ne peuvent mettre en forme seuls, même de façon délirante (ou hallucinatoire).

\section{Interventions double face}

Les interventions double face sont des reformulations qui ont pour vocation d'être des interfaces entre l'individu et le groupe, l'univers interne et le monde externe, le délire et la réalité. Ces interventions visent ainsi à relier au moins deux vertex (Bion, 1965, p. 156), deux points de vue hétérogènes : la réalité psychique partagée du groupe et le monde imaginaire, souvent en partie délirant des patients. Une face de l'intervention est tournée vers le patient, l'autre est tournée vers le clinicien e1 vers le groupe, les deux faces se tolérant mutuellement (en tension) pour progressivement s'articuler et se relier. Ces interventions double face ont, en effet, la fonction d'articulateurs relationnel/émotionnel pour chaque participant et le lien qui les unit. Il s'agit d'organisateur groupalement créé, et porté par le clinicien (fonction phorique, Kaës, 1993), pour le groupe, un organisateur intra et inter-psychique, qui rend possible le nouage, l'organisation et l'intégration des chaînes associatives individuelles dans une chaîne associative groupale (Kaës, 1994) en train de se constituer (Gimenez 2006). Dans toutes ces interventions, le clinicien relève ce qui, pour lui, figure quelque chose qui se passe ici et maintenant, en lui-même et dans le groupe. Les interventions-interprétations trouvées-crées sont celles qui trouvent la forme de ce qui était attendu par le patient (et/ou par le groupe) pour pouvoir le calmer, le réconforter, donner forme et sens à un vécu chaotique et traumatique (Gimenez, 2006). Ces interprétations, dans leur aspect biface, sont porteuses d'un processus de reliaison subjectivante (Pinel, 2011).

Le clinicien, comme être biface, devient, non seulement, un lieu de dépôt, mais aussi de figuration et de transformation: support de la fonction alpha remobilisée chez le patient et dans le groupe. Ces interventions, tout comme des interventions de certains patients, peuvent véritablement avoir un effet symboligène, contenant et permettent une intégration de la chaîne associative du groupe. Les patients sont alors vraiment présents et à l'écoute : d'eux-mêmes, des autres et du groupe, présents à la souffrance et l'histoire des autres, comme si, le temps d'un instant, les processus psychotiques délirants et hallucinatoires passaient au second plan.

\section{Ne pas imposer la violence de la nécessité du sens}

La pratique de ce type de groupe nous a enseigné qu'il était nécessaire de fonctionner en même temps à un second niveau : laisser chacun de ces éléments indépendants, sans lui imposer la violence de la nécessité du sens, de la signification, du lien. Le clinicien apprend ainsi à laisser se poser en lui, à la fois les mots, phrases, expressions successives et les charges émotionnelles qui leur sont liées, mais aussi sans vouloir les insérer nécessairement dans une trame de sens et de signification organisée et sans essayer de s'en débarrasser, quand il ne les comprend pas où quand elle sont trop crues ou trop chargées.

\section{L'attaque de la signification}

Le troisième niveau est d'autant plus présent que le deuxième n'est pas respecté : le sens et le lien qui commence à se construire peut être, dans certains cas, violement attaqué, mis en défaut, mis en pièce, détruit, délié. Nous faisons l'hypothèse que ce troisième niveau, du négatif, mouvement de l'anti-chaîne associative, est présent dans tous les 
groupes, quelle que soit la pathologie des sujets en présence. Il s'agit du travail de déliaison, d'attaque contre les liens, de dissociations, de la destruction du sens et/ou de la signification, de la pulsion de mort ou du travail du négatif. On peut ainsi repérer, dans ce groupe (comme peut-être dans tout groupe), un double mouvement (« associatif-dissociatif ») de « recherche » de figuration, de mise en forme (et de mise en sens), d'une part et, d'autre part, un mouvement d'attaque, de mise en pièce, de destruction de cette mise en forme, eu égard aux tensions, conflits, douleurs de confrontation à la réalité, qu'elle est susceptible de provoquer.

\section{Chaîne associative groupale}

C'est ainsi que commence à se construire une chaîne associative groupale. Les souffrances individuelles (et les traumas qui leurs sont liés), d'abord muettes, sont progressivement réactivées par les associations de chaque-un, et organisées autour des questions de la séparation-arrachement à la mère, de la souffrance crue, de la maltraitance extrême et impensable, de l'abandon, de la trahison, de la haine et la destructivité extrême de l'autre et de soi-même, et de la mort. Toutes les questions contre lesquelles luttent les défenses psychotiques (Gimenez, 2000). Les participants qui utilisaient des figurations délirantes pour s'approcher des formes intolérables à exprimer, et pour figurer en partie l'indicible, se centrent sur une émotion commune et en partie partagée, et semblent participer à tâtons à la construction d'un scénario commun. C'est alors que le vécu, la douleur, la souffrance, la parole et l'expérience de chacun peuvent être écoutées et entendues, trouver leur place et être reçues dans le groupe et en chacun (Gimenez, 2003). Le clinicien, traducteur d'affects de l'un ou des uns pour l'autre (ou pour les autres), investi dans et par le groupe, permet aux interlocuteurs d'explorer, filtrer, réguler, et reconnaître, sans trop de risque extrême, ce qui était là, souvent en négatif et mobilisé par la dynamique de la relation (Gimenez, 2006).

Le groupe, qui est souvent lieu d'expression inorganisée d'expressions délirantes imprégnées de souffrance crue, devient le lieu de partage d'une pensée, un lieu d'accueil pour une chaîne associative groupale enfin possible, et la psyché de chaque sujet peut enfin s'appareiller et s'accorder. Le travail psychique, qui pouvait sembler individuellement trop risqué au-dedans, est groupalisé, mis en commun et externalisé dans l'espace du groupe. Ce qui était intolérable en chacun est ainsi exploré sans trop de risque dans le groupe, dans l'autre et à travers les autres, dans une enveloppe suffisamment protectrice et dans un mouvement de co-création groupale. Dans le groupe, peut alors se développer, par l'apport de chacun, et par l'accompagnement du double mouvement associatif-dissociatif, le travail de transformation introjective de ce qui était, pour chacun, impensable, chaotique et informe.

\section{RÉFÉRENCES}

Avron (Ophélia).- La pensée scénique. Groupe et psychodrame, Toulouse, Érès, 1996.

AnZIEU (Didier).- Les signifiants formels et le Moipeau, dans Anzieu (D.), Doron (D.), Houzel (D.), Misserand (A.), Enriquez (M.), Anzieu (A.), Guillaumin (J.), Lecourt (E.), Nathan (T.), Les enveloppes psychiques. Paris, Dunod, 1987, p. 1-22.

Bion (Wilfred Ruprecht).- Transformations, Paris, Presses universitaires de France, 1965.

BION (Wilfred Ruprecht).- L'attention et l'interprétation, Paris, Payot, 1970.

Casement (Patrick).- À l'écoute du patient, Paris, Presses universitaires de France, 1985.

Gimenez (Guy).- La groupalité psychique dans la thérapie individuelle de schizophrènes, Revue française de psychothérapie psychanalytique de groupe, 27, 1996, p. 109-119.

Gimenez (Guy).- Clinique de l'hallucination psychotique, Paris, Dunod, 2000.

Gimenez (Guy).- Les objets de relation dans la thérapie individuelle et groupale de patients schizophrènes, Revue de psychothérapie psychanalytique de groupe, 41, 2003, p. 41-62.

Gimenez (Guy).- La construction d'une chaîne associative groupale dans le travail psychanalytique avec les patients psychotiques en groupe : du chaos dissociant à une possible polyphonie, Revue de psychothérapie psychanalytique de groupes, 47, 2006, p. 79-91.

KAËs (René).- Le groupe et le sujet du groupe, Paris, Dunod, 1993.

KAËs (René).- La parole et le lien. Processus associatifs dans les groupes, Paris, Dunod, 1994.

KAËs (René).- Les théories psychanalytiques du groupe, Paris, Presses universitaires de France, 1999.

KLEIN (Melanie).- Notes sur quelques mécanismes schizoïdes, dans Klein (M.), Heimann (P.), Isaac (S.), Rivière (J.), Développements de la psychanalyse, Paris, Presses universitaires de France, 1946, p. 274-300.

PINEL (Jean-Pierre).- Group analytic work with violent preadolescents: working through and subjectivation, Group analysis, 44, 2, 2011, p. 196-207. 